\title{
EDITORIAL
}

IMAGING

\section{Imaging in oncology: lighting the way}

$\mathrm{O}$ ver a 100 years ago, the German surgeon Franz König used X-rays to visualize a tumour. Since then, imaging has become an indispensable tool in oncology clinical practice and cancer research. Over the past three decades, enormous strides have been made in the development of imaging technologies and their applications, which have been possible thanks to the collaboration between oncologists, chemists, physicists and engineers in interdisciplinary centres across the world. This expansion in imaging techniques has had a radical impact on the way we diagnose and treat cancer. In this Focus Issue, we have attempted to cover most aspects of the exciting field of oncology that is cancer imaging, including the analysis of innovative techniques, how to assess the utility of these techniques through clinical trials, the challenges of visualizing special tissues, and the use of imaging in cancer beyond detection and response assessment.

Undeniably, imaging has a crucial role in the quest for early detection of all types of cancer. Revolutionary techniques, such as PET, have enabled us to identify small tumours and metastases that would not be visible with any other technique, by detecting their metabolic activity. Andrew C. Peet and colleagues discuss PET and other imaging methods-such as perfusion imaging and magnetic resonance spectroscopy, which also measure tumour metabolism - not only for diagnosis, but also for assessing tumour type, aggressiveness and prognosis in patients with brain tumours.

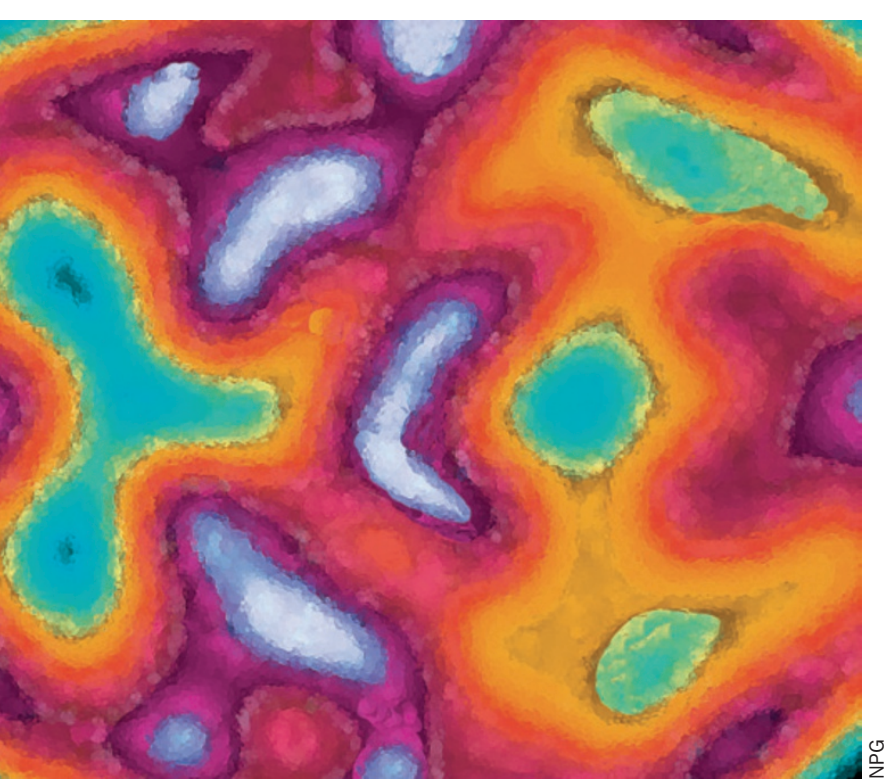

In addition to detection, imaging has a fundamental role in the assessment of whether a tumour is responding favourably to the treatment administered. Image-guided radiotherapy (IGRT) has undergone a remarkable transformation in the past decade through innovative technology that enables daily imaging to locate the tumour precisely, so that the patients can be subsequently repositioned to receive radiation exclusively in the target organ. The exciting advances in IGRT to achieve patient-specific radiotherapy treatment are discussed by David A. Jaffray. In addition, Michael Horsman and colleagues examine the potential use of techniques in routine clinical use to identify hypoxic tumours - which are resistant to radiation treatment-to improve the effectiveness of radiotherapy. For example, PET tracers, especially the nitroimidazoles, can directly identify the presence of hypoxia. Olivier Rouvière and colleagues examine other potential uses of imaging in cancer treatment by discussing highintensity focused ultrasound (HIFU) ablation, which has provided interesting results in the whole-gland treatment of prostate cancer.

However, with the excitement of these developments in recent years come multiple challenges, and several articles in this Focus concentrate on discussing these issues. How can we ensure that we are maximizing the potential of these techniques, and that we are using them effectively and consistently? Are these new technologies within the reach of every cancer centre? Rodney J. Hicks and Michael S. Hofman analyse the efficiency, availability, and cost-effectiveness associated with the tomographic imaging techniques PET-CT and SPECT-CT, and the regulatory hurdles to introducing new tracers that could widen the availability of PET-CT. In turn, Lalitha K. Shankar discusses how assessing the utility of imaging techniques and imaging biomarkers through clinical trials is not an easy task, and describes the efforts of the National Cancer Institute (NCI) in examining imaging modalities and agents to improve image-guided interventions and disease monitoring in cancer.

As concluded in the Timeline by Bhuey Sharma and colleagues, imaging will continue to remodel the landscape of modern oncology, and light the way forward.

All of the Focus articles can be accessed for free until the end of February 2013 at www.nature.com/ nrclinonc/focus/imaging, thanks to support from Siemens Healthcare.

doi:10.1038/nrclinonc.2012.208
M. Teresa Villanueva is the Associate Editor of Nature Reviews Clinical Oncology.

Competing interests The author declares no competing interests. 